\title{
The Efficiency of Radicals Production by Positive Streamer in Air: The Role of Laplacian Field
}

\author{
Andrei A. Kulikovsky, Member, IEEE
}

\begin{abstract}
Two-dimensional modeling of positive streamer in the air between a point anode and a plane cathode is performed. $A$ number of radicals (excited molecules and atoms) produced by a streamer is calculated. The streamers near sharp and dull anodes are simulated. The sharp anode has five times smaller radius of tip curvature, and three times higher Laplacian field at the tip than the dull one. The results show that near the sharp anode, the streamer produces radicals ten times faster and at lower energy cost than near the dull anode. The mean energy cost of one radical is $10 \mathrm{eV}$.
\end{abstract}

Index Terms-Plasma cleaning, streamer breakdown, streamer corona.

\section{INTRODUCTION}

$\mathbf{T}$ HE USE of streamer discharge in plasma chemistry has been a subject of numerous works during the past 20 years. Positive streamer is a bright plasma filament, which propagates between overvolted electrodes. One of the electrodes can be sharpened, thus, the high Laplacian field is then concentrated near that electrode. The streamer is originated in a high field, and then propagates toward the other electrode, where the field is low.

Such a geometry (e.g., wire anode and coaxial tube cathode) is usually used in pulsed streamer corona devices [1]-[3]. It produces multiple streamers in a large discharge gap, applying voltage pulses of relatively low amplitude (10-100 kV). The variants include dielectric barrier discharge, when a thin dielectric covers one of the electrodes, or a discharge in a space filled with dielectric pellets [4].

In all these cases, the chemical reactions in a gas are induced by species generated in the streamer head. The electrons are shifted with respect to ions in the head, and the resulted polarization field facilitates production of new electrons which destroy and excite molecules. The physics of the streamer discharge has been investigated since Rogowski [5] discovered the effect in 1928. The energy of electrons in the streamer head is about $10 \mathrm{eV}$, which is optimal for molecules excitation and dissociation. While moving, the streamer head produces both atoms and excited species. These chemically active species (radicals) then initiate multiple chemical reactions in a gas, leading to removal of harmful components.

Recent experiments on gas cleaning by means of pulsed streamer discharge showed, however, low efficiency of streamer

Manuscript received November 4, 1999; revised December 6, 2000.

The author is with the Department of Chemistry, Carleton University, Ottawa, ON K1S 5B6, Canada, on leave from the Research Computing Center, Moscow State University, 119899 Moscow, Russia (e-mail: akul@srcc.msu.su).

Publisher Item Identifier S 0093-3813(01)02895-8. corona. According to [6] in pure $\mathrm{N}_{2}$, the energy cost of one $\mathrm{N}$ atom production by means of streamer corona is about $300 \mathrm{eV}$. Currently, this technology is more expensive than the electron beam processing, when radicals are generated in a gas by electron beam. The cost of $\mathrm{N}$ atom in pure $\mathrm{N}_{2}$ irradiated with the beam is about $80 \mathrm{eV}$ [6].

The efficiency of streamer corona, however, depends on a number of factors, such as the geometry of electrodes, voltage pulse parameters, gas pressure, etc. In the past five years, two-dimensional models of streamer in nonuniform fields in air [7]-[11] and flue gas [12] have been developed. These models allow one to simulate streamer dynamics and the generation of species, and to investigate the influence of external parameters on species production. The present work is devoted to investigation of the influence of the Laplacian field near the anode, where streamer is generated, on efficiency of radicals generation.

\section{THE MODEL}

\section{A. The Model of Streamer in Air}

Three types of charged species arise in the air under the action of a high electric field: 1) electrons; 2) positive ions; and 3 ) negative ions. The properties of streamer plasma are determined by the number densities of these species $n_{e}, n_{p}$, and $n_{n}$, respectively, and by an electric field $\mathbf{E}$. Neither the current in streamer, nor the rate of field variation can produce significant magnetic field. The electric field is, hence, described by the Poisson equation for electric potential.

The mass of ions (both positive and negative) is much larger than that of the electron. It allows to neglect ions motion in the course of streamer propagation. This assumption is valid for short (about several centimeters) streamers. For long streamers, when time of streamer propagation is larger than $100 \mathrm{~ns}$, the motion of ions may be essential.

Physically, the most important region of streamer is its head, where high field arises due to separation of charges. One may usually assume that the length of electron energy relaxation is much less than the characteristic space scale of field variation. It means that the electron energy distribution function (EEDF) is in equilibrium with the local value of reduced field $E / n$ ( $n$ being the number density of molecules). This local field approximation is very essential, as it gives way to use hydrodynamics for description of electron gas. It also allows one to calculate EEDF and rate constants of electron-molecules reactions for various $E / n$ in advance, and then to use proper values in streamer simulation, or to use experimentally determined dependencies of these rate constants on $E / n$. 
Streamer dynamics are described by the following system of equations:

$$
\begin{aligned}
\frac{\partial n_{e}}{\partial t}+\frac{1}{r} \frac{\partial\left(r j_{e r}\right)}{\partial r}+\frac{\partial j_{e z}}{\partial z} & =S_{\mathrm{ph}}+S_{i}-S_{\mathrm{att}}-L_{\mathrm{ep}} \\
\frac{\partial n_{p}}{\partial t} & =S_{\mathrm{ph}}+S_{i}-L_{\mathrm{ep}}-L_{\mathrm{pn}} \\
\frac{\partial n_{n}}{\partial t} & =S_{\mathrm{att}}-L_{\mathrm{pn}} \\
\Delta V & =-\frac{e}{\varepsilon_{0}}\left(n_{p}-n_{e}-n_{n}\right)
\end{aligned}
$$

where

$$
\mathbf{j}_{e}=-D_{e} \nabla n_{e}-\mu_{e} \mathbf{E} n_{e}
$$

is electron flux, $\mathbf{E}$ is electric field strength: $\mathbf{E}=-\nabla V, V$ potential, $n$ is number density, and $D$ and $\mu$ are diffusion coefficient and mobility, respectively. Subscripts " $e$," " $p$," and " $n$ " refer to electrons, positive, and negative ions, respectively, and " $e$ " in (4) is the absolute value of electron charge.

The right-hand sides of (1)-(3) describe the rates of charged particles production and loss. The $S_{i}$ is the rate of molecules ionization in collision with electrons, $S_{\mathrm{ph}}$ the rate of photoionization in a gas, $S_{\text {att }}$ the rate of electrons attachment to oxygen molecules, $L_{\mathrm{ep}}$ the rate of electron-positive ion recombination, $L_{\mathrm{pn}}$ the rate of positive-negative ions recombination.

The expressions for $S_{i}, S_{\mathrm{att}}, L_{\mathrm{ep}}$, and $L_{\mathrm{pn}}$ are given in [13], [8]. The calculation of the rate of photoionization is based on the model [14], and is described in [15]. The details of numerical solution of system (1)-(4) can be found in [16] and [17]. Boundary conditions for that system are described in [8].

\section{B. Generation of Radicals}

The system (1)-(4), defines the evolution of electron density and electric field in the course of streamer propagation. Knowing these values, one may calculate the number of radicals generated by the streamer in the air.

The number density $n_{a}$ of radicals of a sort $a$ obeys the equation

$$
\frac{\partial n_{a}}{\partial t}=s_{a} k_{a} \xi_{\mathrm{par}} n n_{e}
$$

where

$\xi_{\text {par }} \quad$ fraction of parent molecules $\left(\mathrm{O}_{2}\right.$ or $\left.\mathrm{N}_{2}\right)$ in the air;

$s_{a} \quad$ stoichiometric coefficient;

$k_{a}(E / n)$ rate constant.

In dry air, the most radicals produced are oxygen and nitrogen atoms, and excited nitrogen molecules. The channels of oxygen and nitrogen dissociation, and excitation of nitrogen metastable states are reviewed in [18]. Following [18], the reactions listed in Table I were taken into account. Note that in [8] a reduced set of reactions was used, which led to overestimation of the mean energy cost of each specie (see Section III).

The rate constant of nitrogen dissociation

$$
e+\mathrm{N}_{2} \rightarrow e+\mathrm{N}+\mathrm{N}
$$

TABLE I

List OF RACTIONS AND PARAMETERS OF THEIR RATE CONSTANTS (9) (DATA OF [18]). $\mathrm{N}_{2}(A)$ STANDS FOR $\mathrm{N}_{2}\left(A^{3} \Sigma_{u}^{+}\right), \mathrm{N}_{2}(B)$ FOR $\mathrm{N}_{2}\left(B^{3} \Pi_{q}\right), \mathrm{N}_{2}\left(a^{\prime}\right)$ FOR $\mathrm{N}_{2}\left(a^{\prime 1} \Sigma_{u}^{-}\right)$AND $\mathrm{N}_{2}(C)$ FOR $\mathrm{N}_{2}\left(C^{3} \Pi_{u}\right)$

\begin{tabular}{l|l|l}
\hline Reaction & $A\left(\mathrm{~cm}^{3} \mathrm{~s}^{-1}\right)$ & $B(\mathrm{Td})$ \\
\hline $\mathrm{e}+\mathrm{O}_{2} \rightarrow \mathrm{e}+\mathrm{O}\left({ }^{3} \mathrm{P}\right)+\mathrm{O}\left({ }^{3} \mathrm{P}\right)$ & $10^{-7.9}$ & 134 \\
$\mathrm{e}+\mathrm{O}_{2} \rightarrow \mathrm{e}+\mathrm{O}\left({ }^{3} \mathrm{P}\right)+\mathrm{O}\left({ }^{1} \mathrm{D}\right)$ & $10^{-8}$ & 169 \\
$\mathrm{e}+\mathrm{O}_{2} \rightarrow \mathrm{e}+\mathrm{O}\left({ }^{3} \mathrm{P}\right)+\mathrm{O}\left({ }^{1} \mathrm{~S}\right)$ & $10^{-8.8}$ & 119 \\
\hline $\mathrm{e}+\mathrm{N}_{2} \rightarrow \mathrm{e}+\mathrm{N}_{2}(\mathrm{~A})$ & $10^{-8.4}$ & 140 \\
$\mathrm{e}+\mathrm{N}_{2} \rightarrow \mathrm{e}+\mathrm{N}_{2}(\mathrm{~B})$ & $10^{-8.2}$ & 148 \\
$\mathrm{e}+\mathrm{N}_{2} \rightarrow \mathrm{e}+\mathrm{N}_{2}\left(\mathrm{~W}^{3}, \mathrm{~B}^{\prime}\right)$ & $10^{-8.3}$ & 154 \\
$\mathrm{e}+\mathrm{N}_{2} \rightarrow \mathrm{e}+\mathrm{N}_{2}\left(\mathrm{~B}^{\prime}\right)$ & $10^{-8.7}$ & 168 \\
$\mathrm{e}+\mathrm{N}_{2} \rightarrow \mathrm{e}+\mathrm{N}_{2}\left(\mathrm{a}^{\prime}\right)$ & $10^{-8.8}$ & 167 \\
$\mathrm{e}+\mathrm{N}_{2} \rightarrow \mathrm{e}+\mathrm{N}_{2}\left(\mathrm{a}, \mathrm{w}^{1}\right)$ & $10^{-8.5}$ & 174 \\
$\mathrm{e}+\mathrm{N}_{2} \rightarrow \mathrm{e}+\mathrm{N}_{2}\left(\mathrm{a}^{\prime}\right)$ & $10^{-8.7}$ & 175 \\
$\mathrm{e}+\mathrm{N}_{2} \rightarrow \mathrm{e}+\mathrm{N}_{2}(\mathrm{C})$ & $10^{-8.2}$ & 211 \\
$\mathrm{e}+\mathrm{N}_{2} \rightarrow \mathrm{e}+\mathrm{N}_{2}(\mathrm{E}, \mathrm{a})$ & $10^{-10.1}$ & 254 \\
$\mathrm{e}+\mathrm{N}_{2} \rightarrow \mathrm{e}+\mathrm{N}_{2}(\mathrm{E})$ & $10^{-9.2}$ & 262 \\
\hline
\end{tabular}

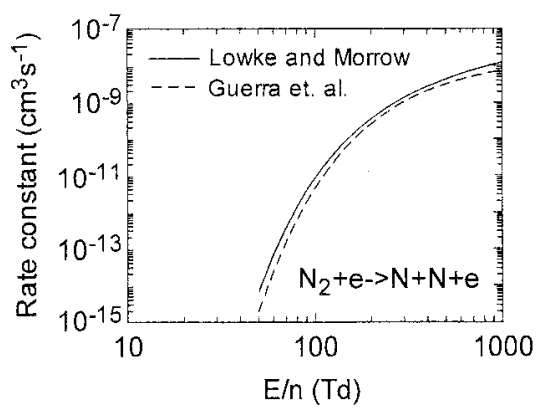

Fig. 1. Nitrogen dissociation rate constant. Solid line: the fit (8) of data [19]; dashed line: the data of [20]

is not given in [18], and was taken from [19]. Lowke and Morrow [19] calculated EEDF and the rate of nitrogen dissociation for the flue gas. The energy of $\mathrm{N}_{2}$ dissociation is rather high, and the rate constant of that process is defined by high-energetic tail of electron energy distribution function, where no vibrational excitation of flue gas molecules occurs. For that reason, ionization coefficients in air and flue gasses are close to each other. One may, therefore, expect, that this is true for the nitrogen dissociation rate constant. We interpolate data [19] with the following expression:

$$
k_{7}=4 \times 10^{-8} \sqrt{\frac{E / n}{2800}} \exp \left(-\frac{680}{E / n}\right) \mathrm{cm}^{3} \cdot \mathrm{s}^{-1}
$$

where $E / n$ is in $\mathrm{Td}\left(1 \mathrm{Td} \equiv 10^{-17} \mathrm{~V} \cdot \mathrm{cm}^{2}\right)$. Fig. 1 shows comparison of the rate constant (8) with the one given in [20]. Both dependencies are in good agreement.

The rate constants of reactions, listed in Table I, have the form

$$
k_{a}=A_{a} 10^{-\left(B_{a} /(E / n)\right)} \quad \mathrm{cm}^{3} \cdot \mathrm{s}^{-1}
$$

where $E / n$ is in Td. The corresponding parameters $A_{a}$ and $B_{a}$ were taken from [18] and are listed in Table I.

\section{RESULTS AND DISCUSSION}

The streamers were simulated in atmospheric pressure air ( $p=760$ torr, $T=300 \mathrm{~K}$ ) in a 2-cm gap between the curved anode and a plane cathode. Cylindrical coordinates with the 


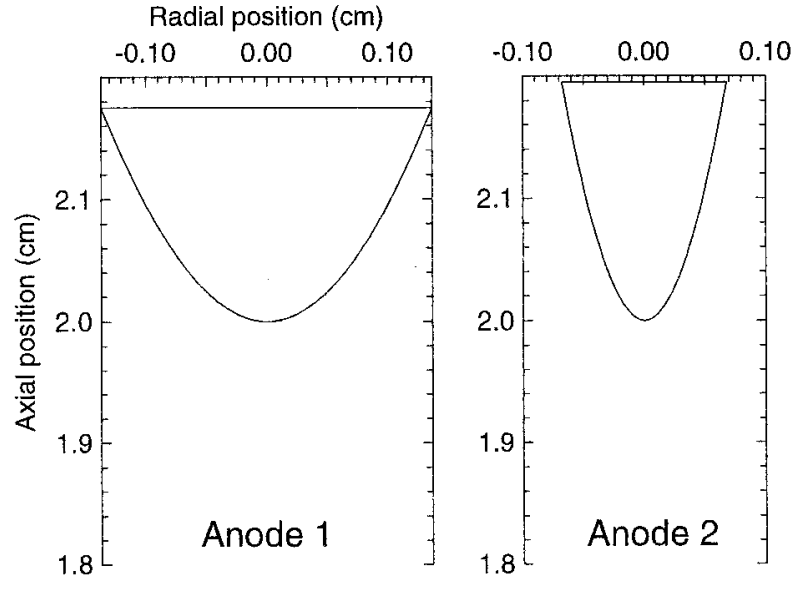

Fig. 2. The two anodes used in simulations.

TABLE II

PARAMETERS OF THE ANODES (10)

\begin{tabular}{l|c|c|c}
\hline & $\mathrm{a}(\mathrm{cm})$ & $\mathrm{b}(\mathrm{cm})$ & $r_{\text {anode }}(\mathrm{cm})$ \\
\hline Anode 1 & 0.32 & 2 & 0.0512 \\
Anode 2 & 0.15 & 2 & 0.0113 \\
\hline
\end{tabular}

origin at the cathode surface opposite to the anode tip were used. The $z$ axis is directed toward the anode tip.

Two anodes were considered: dull (Anode 1) and sharp (Anode 2) (Fig. 2). Both have a form of hyperboloid of revolution

$$
\left(\frac{z}{b}\right)^{2}-\left(\frac{r}{a}\right)^{2}=1
$$

where $a$ and $b$ define the radius of curvature of anode tip: $r_{\text {anode }}=a^{2} / b$. In both cases, $b=2 \mathrm{~cm}$ (the gap distance). Parameters $a$ and $b$, and the radii of tip curvature for both anodes, are given in Table II. The radius of tip curvature of Anode 1 is almost five times higher than that of Anode 2. Under the same applied voltage $(10 \mathrm{kV})$, it gives three times lower Laplacian field near the tip (Fig. 3).

To initiate streamer, the small spot of a seed plasma was placed near the anode tip. In both cases, the seed plasma had the same shape:

$$
\begin{aligned}
\left.n_{p}(r, z)\right|_{t=0} & =\left.n_{e}(r, z)\right|_{t=0} \\
& =n_{0} \exp \left(-\left(\frac{r}{\sigma_{r}}\right)^{2}-\left(\frac{z-z_{0}}{\sigma_{z}}\right)^{2}\right)
\end{aligned}
$$

with $n_{0}=10^{14} \mathrm{~cm}^{-3}, \sigma_{r}=0.01 \mathrm{~cm}, \sigma_{z}=0.02 \mathrm{~cm}$, and $z_{0}=1.985 \mathrm{~cm}$. Initially, there were no negative ions in the gap. In both cases, the applied voltage was $10 \mathrm{kV}$.

It should be emphasized that the only difference between two variants is the shape of the anode. All other parameters, including initial and boundary conditions, remain the same. This allows to elucidate the role of the Laplacian field near the tip in production of radicals.

The streamers for both variants at the moment $5 \mathrm{~ns}$ are shown in Fig. 4. It is seen that near the dull anode, the streamer is narrower and shorter than near the sharp one. The high-field region occupies a much larger volume, in case of a sharp anode.

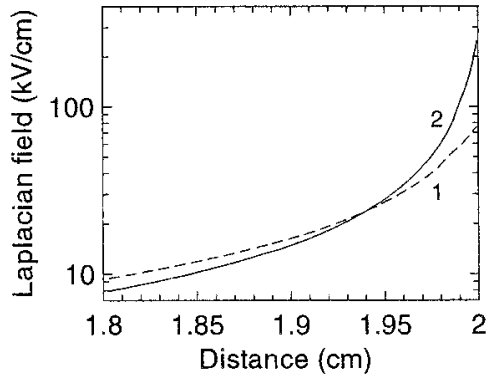

Fig. 3. Axial profiles of Laplacian field near the tip of the anode. Dashed curve: Anode 1. Solid curve: Anode 2. Applied voltage is $10 \mathrm{kV}$ in both cases.
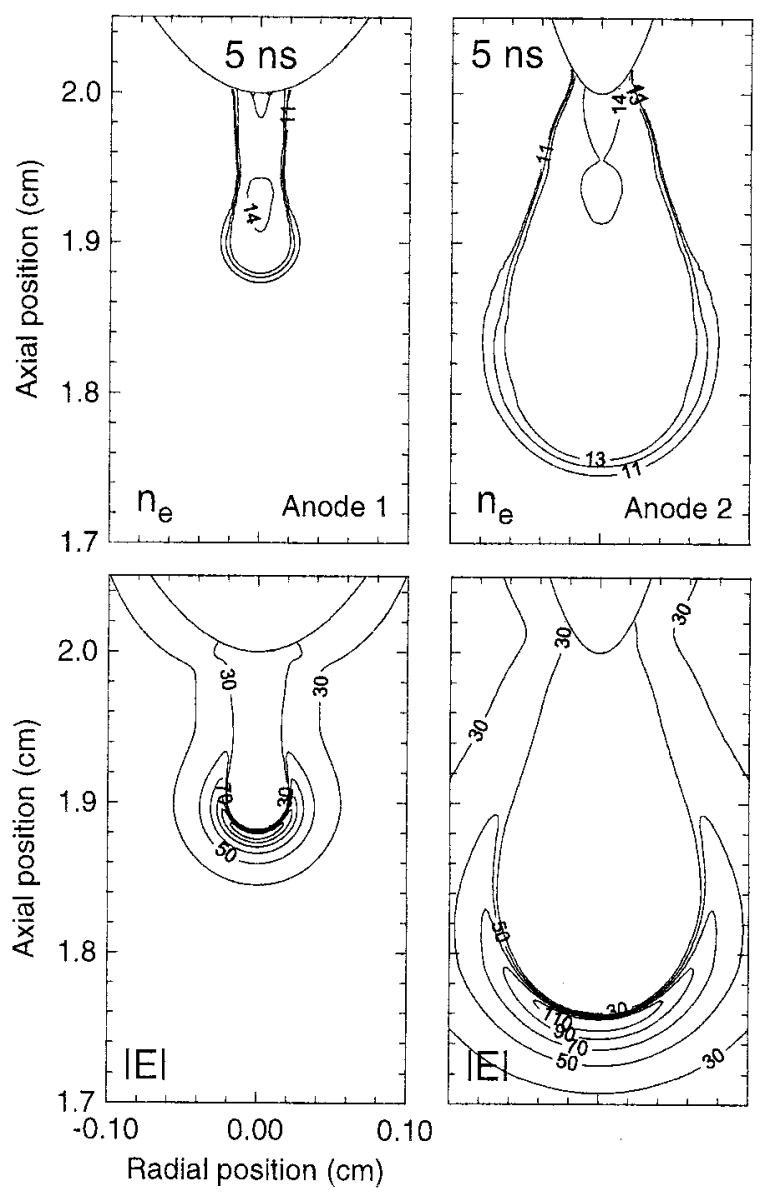

Fig. 4. Contour lines of electron density and absolute value of electric field for the moment $5 \mathrm{~ns}$ for the two anodes. Left pair of maps: Anode 1. Right pair: Anode 2. Top maps: Electron density. Bottom maps: Absolute value of electric field strength. Electron density contours are labeled with the power of 10: $10^{11}$ to $10^{14} \mathrm{~cm}^{-3}$. The outermost contour is $10^{11} \mathrm{~cm}^{-3}$ in both maps. Electric field contours are $30,50,70 \ldots \mathrm{kV} / \mathrm{cm}$.

Fig. 5 shows the contour lines of the rate of nitrogen atoms production (the right hand side of (6) for nitrogen atoms). It is seen, that the streamer near the sharp anode produces $\mathrm{N}$ atoms much faster. This conclusion is also true for the other species. Fig. 6(a) shows the dependence of amount of produced species on time. It is seen that the streamer near the sharp anode produces all radicals almost ten times faster than the streamer near the dull anode. Fig. 6(b) displays the energy cost of the overall amount of species shown in Fig. 6(a). In 1.5 ns, the streamer near the sharp anode becomes more effective than near the dull 


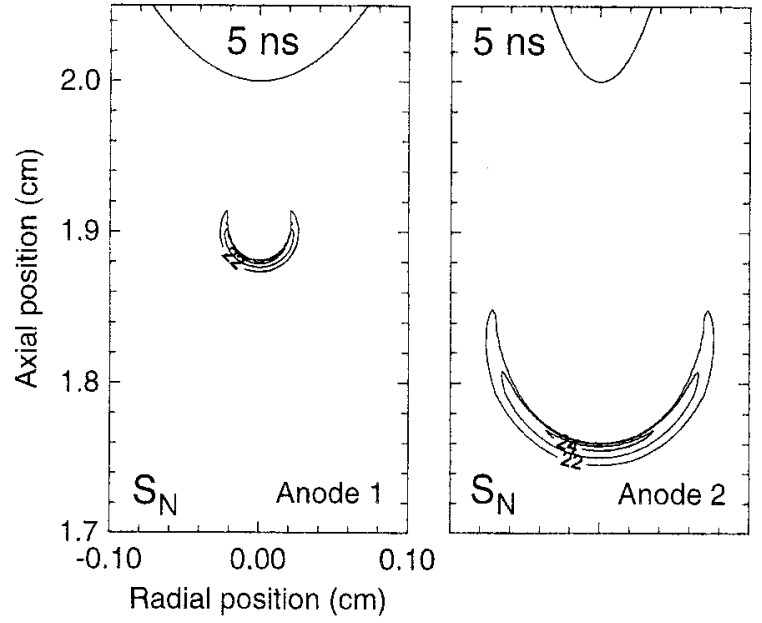

Fig. 5. Contour lines of the rate of $\mathrm{N}$ atoms production at the moment $5 \mathrm{~ns}$ for the two types of anodes. The three contours are shown: $10^{22}, 10^{23}$ and $10^{24}$ $\mathrm{cm}^{-3} \mathrm{~s}^{-1}$.

one. The mean energy cost of one specie for the sharp anode is as low as $10 \mathrm{eV}$.

One must be careful in determining the energy cost of oxygen atoms. To avoid confusion, it should be noted that the cost of the atom is twice as low as the cost of each dissociation event. The number of $\mathrm{O}$ atoms produced in the reactions, listed in Table $\mathrm{I}$, is shown in Fig. 6. The energy cost of each $\mathrm{O}$ atom, at the moment $10 \mathrm{~ns}$, is about $55 \mathrm{eV}$ for Anode 1, and $39 \mathrm{eV}$ for Anode 2. However, in both cases, the most generated active species are excited nitrogen molecules, Fig. 6(a). These molecules are rapidly converted to oxygen atoms in the reaction ${ }^{1}$

$$
\mathrm{N}_{2}^{*}+\mathrm{O}_{2} \rightarrow \mathrm{N}_{2}+\mathrm{O}+\mathrm{O} .
$$

The energy cost, presented in Fig. 6(b) is, from a practical point of view, the cost of an oxygen atom. This should be taken into account when comparing these data with measurements.

The results presented show that the sharp anode has evident advantages over the dull one. Due to a higher Laplacian field, the streamer near the sharp anode is created with a higher head radius; it produces species faster and on time scale $10 \mathrm{~ns}$ the mean cost of each specie is lower.

In [8], and [10], we have reported the values 35 and 30 $\mathrm{eV} /$ specie, respectively, resulting from numerical simulation of streamers in similar conditions. However, in [8] and [10], among possible channels of nitrogen excitation, only production of $\mathrm{N}_{2}\left(A^{3} \sigma\right)$ was taken into account. The set of reactions used here (Table I) takes into account all the channels of nitrogen excitation recommended in [18] which lead to lower energy cost.

A streamer near the sharp anode cannot proliferate far into the low field region. One may assume that it is then transformed into a streamer with smaller radius of head [15]. The effect of the energy cost reduction, therefore, is caused by the high field near the anode. This is in line with the prediction [15], that in low field only the equilibrium streamer can exist, whose properties

${ }^{1}$ For $\mathrm{N}_{2}\left(C^{3} \Pi_{u}\right)$ the rate constant of (12), reported in [18], is $3 \times 10^{-10}$ $\mathrm{cm}^{3} / \mathrm{s}$. Under atmospheric pressure it gives characteristic time of nitrogen de-excitation in reaction (12) about $0.5 \mathrm{~ns}$.

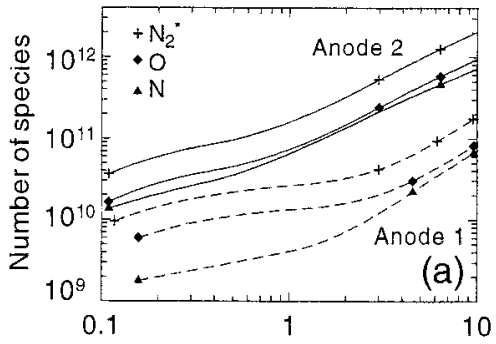

(a)

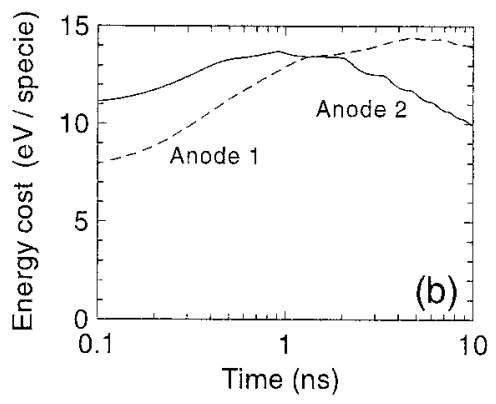

(b)

Fig. 6. (a): Total number of species, produced by the two streamers. Dashed curves: Streamer near dull anode (Anode 1). Solid curves: Streamer near sharp anode (Anode 2). (b): Mean energy cost of each specie for the two streamers. Dashed curve: Anode 1. Solid curve: Anode 2.

depend only on gas pressure, but not on applied voltage and anode geometry. The streamer shown in Fig. 4 (Anode 2), is nonequilibrium in that sense. This is the reason for its better performance.

The presented formulation of the problem assumes that the voltage is applied to the anode instantly. In practice, however, high voltage generators provide pulses with finite rise time, and the formation of streamers also depends on voltage rise time.

Consider a long wire anode. If the voltage rise time is large, most of the streamers will start before the voltage reaches peak value. These streamers grow and propagate initially in the low field, that is they are the "equilibrium" (with a small radius), and have poor efficiency of radicals production. If the voltage rise time is small, the voltage grows faster than the streamer moves, and one may expect formation of nonequilibrium streamers near the anode. Hence, to ensure high performance of species production, it is beneficial to create a high field near the anode as fast as possible, and to interrupt the voltage pulse before the streamers will loose efficiency. Today, the generation of short pulses in large-scale setups is hardly possible. However, such pulses can be generated in a small gaps $(\simeq 1 \mathrm{~cm})$. Besides, the high voltage technology progresses, and one may expect that, in the future, this problem will be solved.

In experiments on streamer corona cleaning, the voltage rise time is usually hundreds of nanoseconds to microseconds [3]. Under such voltage pulses streamers form in the low Laplacian field, and this may be a possible explanation of their low efficiency.

On a time scale of tens of nanoseconds, another essential channel of energy consumption arises: vibrational excitation of nitrogen molecules. The role of vibrationally excited nitrogen molecules in plasma chemistry is not quite clear. Recently, Knizhnik et al. [21] assumed that the energy of vibrational 
excitation is transferred to electronic states (V-E exchange), and electronically excited nitrogen then destroys oxygen in the reactions (12). The measured energy cost of ozone production, [21], was $7 \mathrm{eV} / \mathrm{specie}$, and such a low value was explained by these processes. However, the rate constant of $\mathrm{V}-\mathrm{E}$ conversion is poorly known. On the other hand, it is not clear what the voltage rise time in experiments of [21] was. If it is small enough, the low energy cost may also be attributed to the better streamer efficiency.

Recently, the existing setups used in experiments with pulsed streamer corona cleaning were reviewed [3]. In most cases, the coaxial geometry is used. The diameter of the inner cylinder (anode) is $1.5 \mathrm{~mm}$, or more. The peak Laplacian field at the surface of the inner cylinder is $V /(r \ln (R / r))$, where $R$ and $r$ are radii of outer and inner cylindrical electrodes. The voltage pulses are usually about $20-40 \mathrm{kV}$, therefore, the peak field near the anode is about $100 \mathrm{kV} / \mathrm{cm}$. This corresponds to the conditions near the dull anode described above.

Comparison showed that the better efficiency provides Siemens reactor, where the anode "was structured to obtain a homogeneous distribution of microdischarges ..." [3]. This stricture can provide local enhancement of the Laplacian field. In spite of a very low voltage rise time $(1 \mu \mathrm{s})$, some streamers are born when the field is high. This could be an explanation of better reactor efficiency.

\section{REFERENCES}

[1] M. Tas, “"Plasma-Induced Catalysis. A Feasibility Study and Fundamentals"," Ph.D. dissertation, Eindhoven Univ. Tech., Eindhoven, The Netherlands, 1995.

[2] M. A. Tas, R. van Hardeveld, and E. M. van Veldhuizen, "Reactions of NO in a positive streamer corona plasma," Plasma Chem. Plasma Process., vol. 17, no. 4, pp. 371-391, 1997.

[3] B. M. Penetrante, J. N. Bardsley, and M. C. Hsiao, "Kinetic analysis of nonthermal plasmas used for pollution control," Jpn. J. Appl. Phys., vol. 36, pp. 5007-5017, 1997.

[4] B. M. Penetrante et al., "Comparison of electrical discharge techniques for nonthermal plasma processing of $\mathrm{NO}$ in $\mathrm{N}_{2}$," IEEE Trans. Plasma Sci., vol. 23, pp. 679-687, Aug. 1995

[5] W. Rogowski, "Stoßspannung und durchlag bei gasen," Arch. Electrotech., vol. 20, no. 1, pp. 99-106, 1928

[6] B. M. Penetrante et al., "Identification of mechanisms for decomposition of air pollutants by nonthermal plasma processing," Plasma Sources Sci. Technol., vol. 6, no. 3, pp. 251-259, 1997.

[7] N. Yu. Babaeva and G. V. Naidis, "Two-dimensional modeling of positive streamer dynamics in nonuniform electric fields in air," J. Phys. D, Appl. Phys., vol. 29, no. 9, pp. 2423-2431, 1996.

[8] A. A. Kulikovsky, "Production of chemically active species in the air by a single positive streamer in a nonuniform field," IEEE Trans. Plasma Sci., vol. 25, pp. 439-446, June 1997.
[9] _ - "Positive streamer in a weak field in air: A moving avalanche-tostreamer transition," Phys. Rev. E, Stat. Phys. Plasmas Fluids Relat. In terdiscip. Top., vol. 57, no. 6, pp. 7066-7074, 1998.

[10] _ _ "Analytical model of positive streamer in weak field in air: Application to plasma chemical calculations," IEEE Trans. Plasma Sci., vol 26, pp. 1339-1346, Aug. 1998.

[11] C. E. Georghiou, R. Morrow, and A. C. Metaxas, "The theory of short-gap breakdown of needle point-plane gaps in air using finite-difference and finite-element methods," J. Phys. D, Appl. Phys., vol. 32, no. 12 , pp. 1370-1385, 1999

[12] N. Yu. Babaeva and G. V. Naidis, "Two-dimensional modeling of positive streamer propagation in flue gases in sphere-plane gaps," IEEE Trans. Plasma Sci., vol. 26, pp. 41-44, Feb. 1998.

[13] A. A. Kulikovsky, "Positive streamer between parallel-plate electrodes in atmospheric pressure air," J. Phys. D, Appl. Phys., vol. 30, no. 3, pp. 441-450, 1997.

[14] M. B. Zheleznyak, A. Kh. Mnatsakanian, and S. V. Sizykh, "Photoionization of nitrogen and oxygen mixtures by radiation from gas discharge," High Temp., vol. 20, no. 3, pp. 357-362, 1982

[15] A. A. Kulikovsky, "The role of photoionization in streamer dynamics," J. Phys. D, Appl. Phys., vol. 33, no. 12, pp. 1514-1524, 2000.

[16] _ _ "The structure of streamers in $\mathrm{N}_{2}$. I: Fast method of space-charge dominated plasma simulation," J. Phys. D, Appl. Phys., vol. 27, pp. 2556-2563, 1994.

[17] — "A more accurate Scharfetter-Gummel algorithm of electron transport for semiconductor and gas discharge simulation," J. Comput. Phys., vol. 119, no. 1, pp. 149-155, 1995.

[18] I. A. Kossyi et al., "Kinetic scheme of the nonequilibrium discharge in nitrogen-oxygen mixtures," Plasma Sources Sci. Technol., vol. 1, no. 3 , pp. 207-220, 1992.

[19] J. J. Lowke and R. Morrow, "Theoretical analysis of removal of oxides of sulfur and nitrogen in pulsed operation of electrostatic precipitators,' IEEE Trans. Plasma Sci., vol. 23, pp. 661-671, Aug. 1995.

[20] V. Guerra et al., "Calculated data on electron transport and excitation rate coefficients in $\mathrm{N}_{2}-\mathrm{O}_{2}$ and $\mathrm{N}_{2}-\mathrm{H}_{2}$ discharges," Plasma Sources Sci. Technol., vol. 6, pp. 220-230, 1997.

[21] A. A. Knizhnik et al., "On a possibility of high-efficient ozone production in a streamer discharge," Dokl. Phys., vol. 44, no. 3, pp. 336-339, 1999.

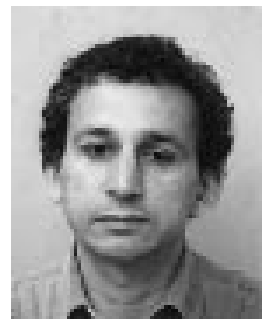

Andrei A. Kulikovsky (M'96) received the M.S. degree in physics from the Moscow Engineering-Physical Institute, Moscow, U.S.S.R., the Ph.D. degree in physics and mathematics from the Institute for High Temperatures, U.S.S.R. Academy of Sciences, Moscow, U.S.S.R., and the D.Sci. degree in physics and mathematics from Moscow State University, Moscow, Russia, in 1974, 1984, and 1998, respectively.

Since 1992, he has been concerned with numerical modeling of streamer corona discharge. In April 1996, he joined the Research Computing Center, Moscow State University, as a Senior Researcher. He is currently a Leading Researcher. His research interests include numerical modeling of physics, chemistry of gas discharges, and the numerical modeling of fuel cells. 